\title{
Advantages, Disadvantages, Indications, Contraindications and Surgical Technique of Laryngeal Airway Mask
}

\author{
Anubhav Jannu, \\ Ashim Shekar, \\ Ramdas Balakrishna, \\ Sudarshan H., \\ Veena G. C., \\ Bhuvaneshwari S. \\ Department of Oral and Maxillofacial \\ Surgery, Rajiv Gandhi University of Health \\ Sciences, Bangalore, India
}

\begin{abstract}
The beauty of the laryngeal mask is that it forms an air tight seal enclosing the larynx rather than plugging the pharynx, and avoid airway obstruction in the oropharynx. The goal of its development was to create an intermediate form of airway management face mask and endotracheal tube. Indication for its use includes any procedure that would normally involve the use of a face mask. The laryngeal mask airway was designed as a new concept in airway management and has been gaining a firm position in anesthetic practice. Despite wide spread use the definitive role of the laryngeal mask airway is yet to be established. In some situations, such as after failed tracheal intubation or in oral surgery its use is controversial. There are several unresolved issues, for example the effect of the laryngeal mask on regurgitation and whether or not cricoids pressure prevents placement of mask. We review the techniques of insertion, details of misplacement, and complications associated with use of the laryngeal mask. We then attempt to clarify the role of laryngeal mask in air way management during anesthesia, discussing the advantages and disadvantages as well as indications and contraindications of its use in oral and maxillofacial surgery.
\end{abstract}

Keywords: Laryngeal / Airway mask / Neck / Intubation

\section{INTRODUCTION}

The laryngeal airway mask (LAM) is a device for anesthetic air way management that received Food and Drug Administration (FDA) approval in 1991 [1]. The mask airway was designed by Brain as a new concept in airway management. The beauty of the laryngeal mask is that it forms an air tight seal enclosing the larynx rather than plugging the pharynx, and avoid airway obstruction in the oropharynx [2].

The goal of its development was to create an intermediate form of airway management face mask and endotracheal tube. Indica-

\footnotetext{
Correspondence: Anubhav Jannu

Department of Oral and Maxillofacial Surgery, Rajiv Gandhi University of Health Sciences, Bangalore, India

E-mail: anubhavjannu@gmail.com

Received Apr 24, 2017 / Revised Sep 18, 2017 / Accepted Dec 7, 2017
}

tion for its use includes any procedure that would normally involve the use of a face mask. The laryngeal mask airway was designed as a new concept in airway management and has been gaining a firm position in anesthetic practice. Despite wide spread use the definitive role of the laryngeal mask airway is yet to be established. In some situations, such as after failed tracheal intubation or in oral surgery its use is controversial. There are several unresolved issues, for example the effect of the laryngeal mask on regurgitation and whether or not cricoids pressure prevents placement of mask.

We review the techniques of insertion, details of misplacement, and complications associated with use of the laryngeal mask. We then attempt to clarify the role of laryngeal mask in air way management during anesthesia, discussing the advantages and disadvantages as well as indications and contraindications of its use in 
oral and maxillofacial surgery [2]. The LMA may offer the oral and maxillofacial surgeon a new airway device for use during general anesthesia in the office [1].

\section{PHYSICAL ROLE OF LARYNGEAL AIRWAY MASK}

The LAM has been used successfully for various surgical procedures in paediatrics, including during dental extraction. Fewer incidence of hypoxia and significantly better arterial oxygen saturation were found with the LAM. Laryngeal mask airway has been the subject of several comprehensive reviews.

The popularity of the LAM stems from its perceived benefits over other airway devices and several studies have shown that overall success rate for the technique and complication rate are low. The LAM, however, is often misused and there is continued debate about frequency of failed placement, critical incidents, such as aspiration of gastric contents, particularly in view of suggestions that the LAM may interfere with lower esophageal sphincter function. Difficulty in viewing the glottis is generally irrelevant for successful LAM placement, making it a useful substitute airway. The curved tube guides the instruments towards the glottis, making it a useful intubation aid. LAM functions both as a ventilator device and as an aid to blind/fiberscope-guided tracheal intubation. Other design goals were to eliminate the need for head \& neck manipulation and insertion of fingers in mouth during placement [3]. Establishing a secure airway in a trauma patient is one of the primary essentials of treatment. Any flaw in airway management may lead to grave morbidity and mortality.

Pressure support ventilation (PSV) is a pressure-targeted mode, which provides breath-by-breath ventilation support, always initiated by patient and synchronized with respiratory effort. It is "gold standard" mode for weaning in mechanically ventilated intensive care patients. PSV has proven to be effective in eliminating the work of breathing (WOB) while also maintaining the patient's spontaneous ventilation. Another advantage of the use of PSV is that it requires less pressure to obtain the target tidal volume than controlled mechanical ventilation. This reduced pressure requirement results in less air leakage during mechanical ventilation with supraglottic airway device like the laryngeal mask air way. In addition, the resulting reduced intrathoracic pressure attenuates the effect of mechanical ventilation on hemodynamic and cardiac output [4]. There is evidence that the cuff of LAM can exert a magnitude of pressure onto the laryngopharyngeal mucosa sufficiently high to cause lingual, hypoglossal or recurrent laryngeal nerve injury, or impede venous and lymphatic drainage [5]. Inspiratory-imposed resistive WOB is a major component of the total WOB for a tracheally intubated, spontaneously breathing patient. Additional resistive work imposed by the breathing apparatus should not be underestimated. The endotracheal tube is a significant resistor in the breathing apparatus. Increased WOB adds to the WOB, which is already increased in diseases such as bronchopulmonary dysplasia. Increased WOB may precipitate respiratory muscle fatigue (respiratory muscles fail as force generators), which leads to hypoventilation, hypercapnia, and hypoxemia. A sensible approach, particularly for those patients predisposed to developing respiratory muscle fatigue, is to decrease the WOB by using either low-flow-resistant equipment, PSV or both. Aspiration as a result of gastroesophagealreflux (GER) and regurgitation remains a serious potential problem in anesthetized patients. Brief pediatric surgical procedures not involving the thorax or abdomen are often performed while the patients are breathing spontaneously, particularly with the widespread use of the LAM. It is therefore clinically relevant to know how anesthesia and resistance added by anesthetic airway apparatuses affect the $\mathrm{WOB}$ in infants and children. An excessive increase in WOB would increase respiratory muscle loading and oxygen consumption, and potentially predispose the patient to respiratory muscle fatigue and failure. Some studies have suggested that LAM reduces WOB compared with endotracheal tube.

It has been hypothesized that anesthesia causes partial upper airway obstruction resulting from pharyngeal muscle relaxation and results in increased WOB and that LAM decreases WOB, as compared with a mask without oral airway. Maxillofacial trauma presents a complex problem with regard to the patients air way [4]. By definition, the injury compromises the patent airway and therefore it must be protected. In most cases, the patients undergo surgery for maxillofacial trauma or for other, more severe, life- 
threatening injuries, and securing the air way is first in the introduction of general anesthesia. In such patients we anticipate difficult endotracheal intubation and, often, also difficult mask ventilation. In addition, the patient is usually regarded as having a "full stomach" and has not been cleared of a C-spine injury, which may complicate airway management furthermore. The time available to accomplish the task is short and the patient's condition may deteriorate rapidly. Both decision-making and performance are impaired in such circumstances. The laryngeal mask has a potential role in patients with difficult airways, including those with limited mouth opening.

\section{DEVICE}

The LAM consists of a triangular mask, the design of which is based on the configuration of hypopharynx. An inflatable cuff is on the inner rim of the mask. The cuff is inflated through a pilot tube with a balloon on the tip, allowing the cuff pressure to be monitored. A tube, which connects the mask to the anesthetic circuit, is fused at a $30^{\circ}$ angle to the back of the mask. A black line along the length of the tube corresponds to the mid-surface of the outer aspect of the mask. Orientation of the black line at both the 12 o'clock position and in the midline of the oral cavity indicates correct positioning of the LAM. Maximizing the laryngeal seal is dependent on obtaining and maintaining the proper seating of the mask within the hypopharynx. The air inlet connecting the tube to the mask has two vertical bars. These bars prevent the epiglottis from lodging against the tube opening and resulting in obstruction of the device. The LAM is available in assorted sizes that will accommodate the infant to the adult. It is made of a soft medical-grade silicone and is designed to be reused after autoclaving. Several modifications of the LAM have been evaluated [1].

\section{TECHNIQUE}

The LAM was designed to form a secure and direct connection with the patient's airway. The standard technique for insertion of the LAM recommends that the patient be in the sniffing position with the neck flexed and the head extended. The cuff should be fully deflated, and the back of the mask slightly lubricated. The inner bowl (mask) of the airway should be free of lubrication because this may either result in irritation of the airway (e.g., laryngospasm) or obstruction of either the respiratory tract or the aperture opening in the LAM. The posterior surface of the mask is pressed against the palate following the curvature of the pharynx and pushed posteriorly into the hypopharynx until firm resistance is felt. In this position, the sides of the mask face into the pyriform fossa, and the upper border of the mask is caudal to the base of the tongue. When the cuff is inflated, a seal is formed around the laryngeal perimeter. Inflation of the cuff results in a slight cephlad displacement of the LAM and a slight bulging of the soft tissues overlying the thyroid and cricoid cartilage. Neuromuscular relaxation is not required for insertion of the LAM. Use of propofol, which provides greater muscular relaxation compared with methohexital, may be advantageous during placement of the LAM.

\section{The standard technique}

(1) The cuff must be fully and correctly deflated before placement. This imparts rigidity to the tip of the cuff. The deflated cuff should be free from wrinkles and it's rim should face away from the mask aperture. This can be achieved by pressing the hollow side down onto a clean flat surface during inflation, with two fingers pressing down on a point just short of the tip.

(2) A lubricant is applied only to the posterior surface of the cuff just before placement. This prevents the cuff tip from rolling over on contact with the palate. Application of lubricant to the anterior surface of the mask is not necessary, and lubricant may block the aperture or be inhaled, causing airway obstruction or coughing.

(3) Before placement, the patient's neck is flexed and the head extended ("sniffing position") by pushing the head from behind with the non-dominant hand (Fig. 1). An assistant should open the mouth by pulling the lower jaw downwards. With experience, the operator can open the mouth with the third finger of the dominant hand.

(4) The device is held between the thumb and the index finger, as close as possible to the junction of the tube and mask. The ap- 
erture of the mask faces the patient's chin.

(5) The tip of the cuff is placed against the inner surface of the patient's upper incisor teeth. It is important that at this point the tube should be parallel to the floor rather than vertical. The mask is then pressed upwards against the hard palate and advanced into the oral cavity, maintaining upward pressure.

(6) The device is advanced using the index finger located at the junction of the tube and the mask. It is essential that the tip of the cuff does not roll over while advancing the laryngeal mask.

(7) A change of direction will be felt as the cuff tip follows the posterior pharyngeal wall downwards. The laryngeal mask is pushed as far as possible into the hypopharynx by the index finger. When the mask is fully advanced, resistance will be felt.

(8) The tube is then held by the non-dominant hand to prevent the mask from moving out of position as the index finger is withdrawn

(9) With experience, the index finger can advance the mask fully into position. However, if it is considered that the mask is not fully advanced into the hypopharynx, the laryngeal mask is pressed downwards by the non-dominant hand for complete location.

(10) The cuff is inflated with an appropriate volume of air. The tube usually moves out of the mouth slightly (mean distance of $0.7 \mathrm{~cm}$ ) [6], and the tissues overlying both the Thyroid and

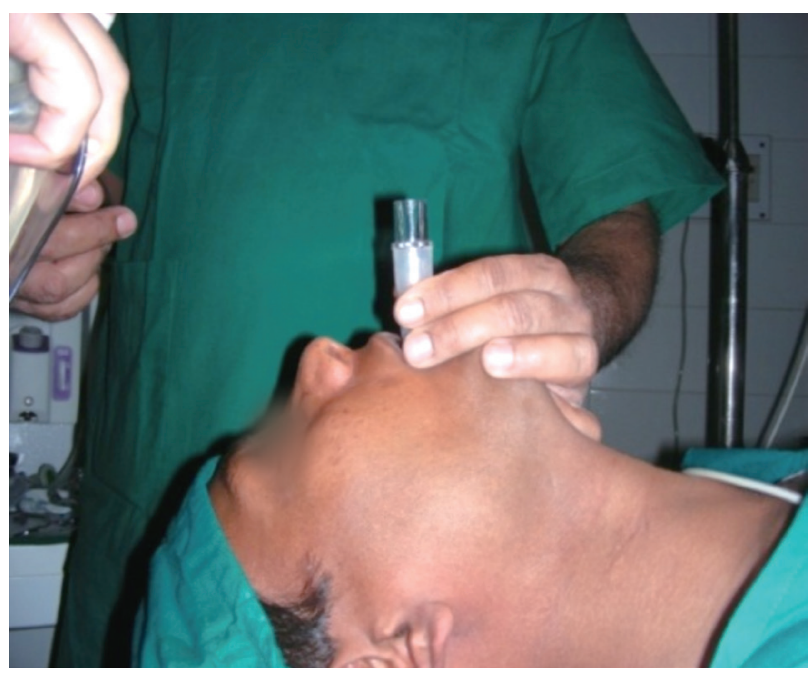

Fig. 1. Insertion of laryngeal airway mask.
Cricoid cartilage bulge slightly when the cuff is inflated. The tube should not be held or connected to the breathing system during inflation. The tip of the mask is likely to be positioned too deeply if the tube is held during inflation.

(11) The laryngeal mask is connected to the breathing system and adequacy of ventilation is assessed (Fig. 2). When adequate ventilation is not obtained, the mask is removed and placement reattempted. The cuff should be prepared between each attempt as initially described.

(12) A bite-block is inserted, and the laryngeal mask and the biteblock are fixed. Brain recommends using a wad of gauze as a bite-block [2].

\section{INDICATIONS}

Patient presents a formidable and unique anaesthetic challenge with several factors contributing to the situation. The foremost concern is that the surgical site within the oral cavity is in close proximity to the pharynx. Performing surgery in the oral cavity therefore renders the patient susceptible to airway obstruction and airway irritation. These factors can result in a significant degree of hypoxia [7,8]. Decreased minute ventilation and airway tone secondary to sedative medications also may contribute to the hypoxia. Although hypoxia is easily recognized with pulse oxim-

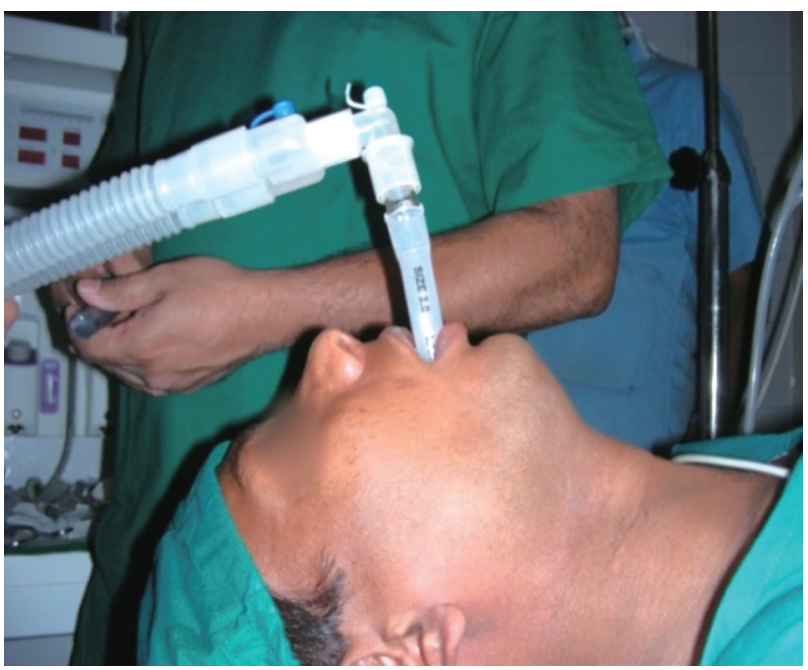

Fig. 2. Connected to artificial airway. 
etry and readily treated, it frequently requires interruption of the surgery and manipulation of the airway. If decreased ventilation occurs with the LAM, assisted or controlled ventilation is possible without interrupting the surgery. The LAM is an excellent barrier against aspiration of saliva and blood within the surgical field $[9,10]$. A pharyngeal curtain is recommended to minimize the possible aspiration of surgical debris (e.g., tooth fragments) when the LAM is removed. However, placement of the pharyngeal curtain does not result in obstruction because the airway is secure. The indications and advantages provided by the LAM are related to its unique connection between the anatomic airway and the artificial airway.

The LAM, with its advantages over both the face mask/nasal hood and endotracheal intubation, potentially has a place in oral and maxillofacial surgery by increasing the safety and efficacy of outpatient general anesthesia in specific situations. Case reports of the LAM for maxillofacial surgery, including adenotonsillectomy, cleft palate repair, and dentoalveolar surgery, have appeared in the literature $[10,11]$. The anesthetic technique used is a modification of that developed using a nasal cannula. Local anesthetic nerve blocks are always used to minimize the general anesthetic requirements. A pharyngeal pack is placed in a manner comparable to traditional method used for outpatient general anesthesia. A wider retractor is seated against the lingual alveolus of the surgical site between the pack and tongue. This protects and minimally displaces the tongue and LAM tube to the opposite side of the mouth.

The LAM also has an important role in acute airway management in the trauma setting or during anesthetic emergencies. Airway control may be established with the LAM when the patient can neither be intubated nor ventilated. In managing the difficult airway, the laryngeal mask airway can be considered before either transtracheal jet ventilation or establishing a surgical airway. After insertion of the LAM, intubation may be attempted, if indicated. A 6.0 or 6.5 endotracheal tube can be passed through a number 3 or number $4 \mathrm{LAM}$, respectively [12]. The $30^{\circ}$ angle at the junction of mask and tube of the LMA facilitates visualization of the glottis during fiberoptic intubation. The intubation is achieved with fiberoptic assistance to avoid damaging the two bars within the ap- erture of the junction between the mask \& tube.

\section{CONTRAINDICATIONS}

There are several contraindications to the use of LAM. It is contraindicated for use in patients who are at an increased risk of gastric aspiration. This list includes the patient with a history of obesity, hiatal hernia, gastroparesis, or trauma. Application of cricoids pressure is often used while obtaining airway control in these atrisk patients. According to Bennett et al. [1] cricoid pressure applied after the insertion of the LAM is effective and does not interfere with LAM ventilation. Bronchospasm, pulmonary edema, or morbid obesity results in high airway resistance or low pulmonary compliance. Inadequate ventilation and gastroesophageal insufflation result with assisted or controlled LAM ventilation. The placement of the LAM is dependent on opening the mouth and passing the airway along the posterior wall of the pharynx. An inability to open the mouth or an infection or pathologic abnormality within the oral cavity or pharynx, can interfere with the use of the LAM.

\section{ADVANTAGES}

The primary advantage of the LAM over the face mask during general anesthesia includes the ability to obtain, secure, and maintain a patent airway. The laryngeal mask airway is passed beyond the tongue, forming a seal with the laryngeal inlet and eliminating the most common cause of upper airway obstruction in the non-intubated patient. Maintenance of a patent airway with fewer episodes of oxygen de-saturation has been demonstrated for the LAM as compared with the face mask [13].

Environmental inhalational gas exposure values associated with the use of a LAM have been shown to be less than those achieved with a face mask and comparable to those with the use of an endotracheal tube. Ocular and facial nerve injuries associated with prolonged face mask use are also avoided. The advantages of the laryngeal mask airway include anesthetic management, induction, maintenance, and emergence. The placement of the LMA can be accomplished without muscle relaxants and laryngoscopy. The 
avoidance of succinylcholine may decrease the incidence of postoperative myalgias. Significant and potentially detrimental hemodynamic changes associated with both laryngoscopy and tracheal intubation are also attenuated and are of shorter duration with the use of the laryngeal mask airway. In our study, we too observed that LAM placement can be accomplished without muscle relaxants. Compared with an endotracheal tube, the anesthetic requirement for tolerance of the LAM has also been reported to be less [14]. Differences in the response to the LAM are also seen during emergence from anesthesia. The LAM is well tolerated, with a lower reported incidence of hyperactive respiratory occurrences (e.g., coughing, laryngospasm, breath holding) than with an endotracheal tube. The anatomic placement of the LAM, with its lack of impingement on the trachea and vocal cords, minimizes complications that are potentially associated with intubation. According to Swann et al. [15] incidence of postoperative sore throat as well as hoarseness is less with the LAM compared with the endotracheal tube.

\section{DISADVANTAGES}

According to Bennett et al. [1] the primary disadvantage and greatest concern with the use of the LAM is the inability to isolate the airway and to protect against the risk of aspiration. Indeed, the LAM has been shown to form a direct conduit between the laryngeal inlet and esophagus by enclosing both. Regurgitation and aspiration have been reported with the use of the LAM. The incidence of regurgitation [16] associated with the use of the LAM varies from $0 \%$ to $23 \%$, which is comparable to the incidence of regurgitation associated with general anesthesia administered by other techniques [17]. However, much of the literature concerning regurgitation and aspiration with the LAM is now recognized to be of questionable scientific design.

These early reports were most likely inflated because of variables such as poor patient selection and patient position during the procedures. If emesis is observed earlier, and in lesser quantity, intervention can be instituted earlier, thus reducing the chance of complications.

\section{CONCLUSION}

So we conclude with suggestion that it can be used in cases which require short surgical procedures under general anesthesia or sedation. The true features and role of the laryngeal mask will be established only through more studies in which the device is used correctly.

\section{CONFLICT OF INTEREST}

No potential conflict of interest relevant to this article was reported.

\section{PATIENT CONSENT}

The patient provided written informed consent for the publication and the use of their images.

\section{REFERENCES}

1. Bennett J, Petito A, Zandsberg S. Use of the laryngeal mask airway in oral and maxillofacial surgery. J Oral Maxillofac Surg 1996;54:134651.

2. Asai T, Morris S. The laryngeal mask airway: its features, effects and role. Can J Anaesth 1994;41:930-60.

3. Brain AI, Verghese C, Addy EV, Kapila A. The intubating laryngeal mask. I: Development of a new device for intubation of the trachea. Br J Anaesth 1997;79:699-703.

4. Dorges V, Ocker H, Wenzel V, Schmucker P. The laryngeal tube: a new simple airway device. Anesth Analg 2000;90:1220-2.

5. Goldmann K, Dieterich J, Roessler M. Laryngopharyngeal mucosal injury after prolonged use of the ProSeal LMA in a porcine model: a pilot study. Can J Anaesth 2007;54:822-8.

6. Leader GL. Facilitation of the insertion of the laryngeal mask. Anaesthesia 1991;46:987.

7. Allen NA, Rowbotham DJ, Nimmo WS. Hypoxaemia during outpatient dental anaesthesia. Anaesthesia 1989;44:509-11.

8. Bone ME, Galler D, Flynn PJ. Arterial oxygen saturation during general anaesthesia for paediatric dental extractions. Anaesthesia 1987;42:879-82.

9. Webster AC, Morley-Forster PK, Dain S, Ganapathy S, Ruby R, Au A, et al. Anaesthesia for adenotonsillectomy: a comparison between tracheal intubation and the armoured laryngeal mask airway. Can J Anaesth 1993;40:1171-7. 
10. Young TM. The Laryngeal Mask in dental anaesthesia. Eur J Anaesthesiol Suppl 1991;4:53-9.

11. Beveridge ME. Laryngeal mask anaesthesia for repair of cleft palate. Anaesthesia 1989;44:656-7.

12. Krausz AA, El-Naaj IA, Barak M. Maxillofacial trauma patient: coping with the difficult airway. World J Emerg Surg 2009;4:21.

13. Pennant JH, Pace NA, Gajraj NM. Role of the laryngeal mask airway in the immobile cervical spine. JClin Anesth 1993;5:226-30.

14. Sarma VJ. The use of a laryngeal mask airway in spontaneously breathing patients. Acta Anaesthesiol Scand 1990;34:669-72.

15. Swann DG, Spens H, Edwards SA, Chestnut RJ. Anaesthesia for gynaecological laparoscopy: a comparison between the laryngeal mask airway and tracheal intubation. Anaesthesia 1993;48:431-4.

16. Morse Z, Sano K, Kageyama I, Kanri T. The relationship of placement accuracy and insertion times for the laryngeal mask airway to the training of inexperienced dental students. Anesth Prog 2002;49:9-13.

17. John RE, Hill S, Hughes TJ. Airway protection by the laryngeal mask; a barrier to dye placed in the pharynx. Anaesthesia 1991;46:366-7. 